\title{
Personalia
}

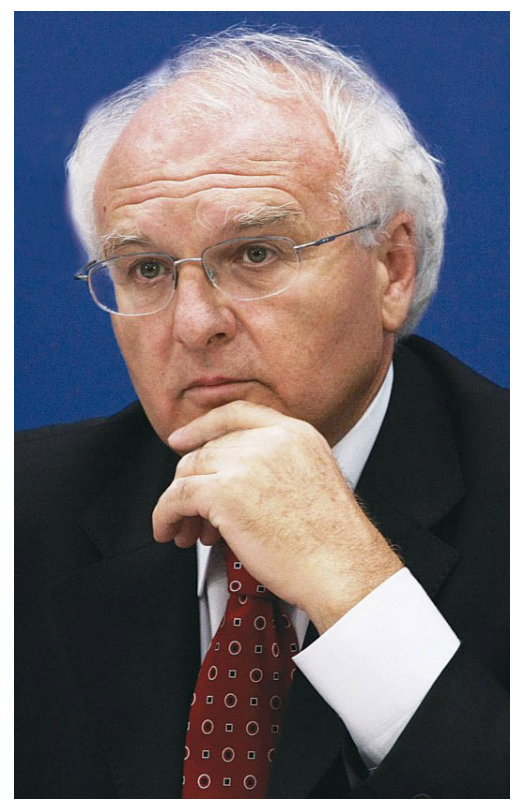

\section{Laudation on the occasion of the 70th anniversary of Ivan Vakarchuk}

Ivan Vakarchuk, a leading Ukrainian physicist, Minister of Education and Science of Ukraine (20072010), Rector of Ivan Franko National University of Lviv (1990-2007, 2010-2013) is celebrating his 70th anniversary on March 6, 2017.

Ivan Vakarchuk was born in the village of Stari Bratushany (now Edineţ district, Moldova). He graduated from the Faculty of Physics of Ivan Franko State University of Lviv in 1970, where in 1973 he also earned his PhD in physics under the supervision of Ihor Yukhnovskii. His PhD thesis "Application of the method of displacements and collective variables in the study of interacting Bose particles near absolute zero" was followed in 1980 by defending his habilitation thesis "Microscopic theory of the Bose liquid" and becoming one of the youngest doctors habilitated in physics.

Prof. Vakarchuk is well regarded for his numerous important contributions to several diverse areas of theoretical physics, including statistical physics, fundamental problems of quantum mechanics and quantum information, mathematical methods of theoretical physics, general relativity, cosmology and theory of stellar spectra, and geophysics. In statistical physics, his favorite and main field of expertise and the subject of his life-long passion, the major results of Vakarchuk are in the theory of quantum liquids such as superfluid helium-4, theory of phase transitions and critical phenomena, physics of disordered and magnetic systems. Today, scientific achievements of Vakarchuk are reflected in about 400 publications.

In the 1970s, Vakarchuk built a quantitative theory of the Bose-Einstein condensation in superfluid He- 4 based on the density matrices method. In the 1980s, using the functional integration formalism, he developed a chain of functional derivative equations for calculation of correlation functions of both classical and quantum many-body systems. At the same time, he became interested in astrophysics working on the quantum theory of radiative transfer in stellar atmospheres and on different mechanisms for broadening the atomic spectral lines.

In the 1980s and 1990s, Vakarchuk co-authored an extensive series of articles concerning the theory of disordered and magnetic systems. He studied the phonon, spin, and electron excitations in the systems with structural disorder, as well as an impact of the external magnetic field on the Bose-Einstein condensation. 
Since the end of 1990s, Vakarchuk and his co-workers from the Department for Theoretical Physics, University of Lviv, pioneered in Ukraine the research in the area of quantum mechanics with deformed Heisenberg algebra, as well as quantum information. At the beginning of 2000s, he proposed a new method for analytical calculation of expressions for density matrix and thermodynamic functions of quantum liquids that are valid in a wide range of temperatures including the $\lambda$-transition. In recent years, Vakarchuk has continued his studies of impurity states in many-boson systems.

Vakarchuk is an inspiring teacher, keen popularizer of science and original thinker, and effective manager. He has trained several generations of scientists: $17 \mathrm{PhD}$ and 3 habilitation projects have been successfully defended under his supervision. His manuals and textbooks "Lectures on general relativity", "Introduction to the many-body problems", "The theory of stellar spectra", and "Quantum mechanics" (4 editions) have become classics among students and professionals, the latter having been awarded the State Prize of Ukraine in Science and Technology in 2000. Vakarchuk is a founder and editor-in-chief of journals "Journal of Physical Studies" and "World of Physics", member of the editorial boards of the journals "Condensed Matter Physics" and "Bulletin of Lviv University. Physical Series". He was behind the rekindling of the Lviv-Warsaw school of philosophy of science after a half-a-century halt. He headed the Department of quantum statistics at Lviv statistical physics division of the Institute for Theoretical Physics, affiliated with the Academy of Sciences of the Ukrainian SSR (1980-1984, now: Bogoliubov Institute for Theoretical Physics of the National Acad. Sci. of Ukraine) and the Department for Theoretical Physics (1984-2015) of Ivan Franko National University of Lviv. As a Rector of Ivan Franko National University of Lviv, he was a fervent promoter of the idea of the universal nature of education at universities, paying special attention to links between natural sciences and humanities. It was during his office as a Rector that the University was awarded the title of "National" and was significantly expanded to include new faculties and new departments. As Minister of Education and Science of Ukraine, he made a major contribution into successful launching of the External Independent Testing, a universal examination for admission to universities in Ukraine.

The Editorial board, fellow physicists and students are wishing to Professor Vakarchuk to stay in good health, the joy of family and friends, and many more fruitful ideas, scientific results and inspiring discussions bringing his inquisitive universal mind closer to the scientific truth.

Ad multos annos! 